\title{
The Hypothesis of Subliminal Cue Reactivity in Addiction Revisited: An fMRI Study
}

\author{
Joar Guterstam ${ }^{\text {a }}$ Nitya Jayaram-Lindström ${ }^{a} \quad$ Jonathan Berrebi ${ }^{b}$ \\ Predrag Petrovic ${ }^{a, b}$ Martin Ingvar ${ }^{b}$ Peter Fransson ${ }^{b}$ Johan Franck $^{a}$ \\ aDepartment of Clinical Neuroscience, Centre for Psychiatry Research, Karolinska Institutet, \& Stockholm \\ Health Care Services, Stockholm County Council, Stockholm, Sweden; bepartment of Clinical Neuroscience, \\ Karolinska Institutet, Stockholm, Sweden
}

\section{Keywords}

Cue reactivity - Subliminal - Stimulant use disorder .

Functional magnetic resonance imaging

\begin{abstract}
Introduction: Exposure to conditioned cues is a common trigger of relapse in addiction. It has been suggested that such cues can activate motivationally relevant neurocircuitry in individuals with substance use disorders even without being consciously perceived. We aimed to see if this could be replicated in a sample with severe amphetamine use disorder and a control group of healthy subjects. Methods: We used fMRI to test the hypothesis that individuals with amphetamine use disorder, but not healthy controls, exhibit a specific neural reactivity to subliminally presented pictures related to amphetamine use. Twenty-four amphetamine users and 25 healthy controls were recruited and left data of sufficient quality to be included in the final analysis. All subjects were exposed to drug-related and neutral pictures of short duration (13.3 ms), followed by a backward visual mask image. The contrast of interest was drug versus neutral subliminal pictures. Results: There were no statistically significant differences in BOLD signal between the drug and neu-
\end{abstract}

karger@karger.com www.karger.com/ear

Karger!
C 2022 The Author(s).

Published by S. Karger AG, Basel

This article is licensed under the Creative Commons Attribution 4.0 International License (CC BY) (http://www.karger.com/Services/ OpenAccessLicense). Usage, derivative works and distribution are permitted provided that proper credit is given to the author and the original publisher. tral cues, neither in the limbic regions of primary interest nor in exploratory whole-brain analyses. The same results were found both in amphetamine users and controls. Discussion/ Conclusion: We found no evidence of neural reactivity to subliminally presented drug cues in this sample of subjects with severe amphetamine dependence. These results are discussed in relation to the earlier literature, and the evidence for subliminal drug cue reactivity in substance use disorders is questioned.

(C) 2022 The Author(s) Published by S. Karger AG, Basel

\section{Introduction}

Craving for drugs serves as a diagnostic criterion for substance use disorders in both DSM-5 and ICD-10 and is a central concept in most theoretical models of addictive disorders [1]. It is strongly linked to relapse in drugseeking behavior and serves as an important target in both psychological and pharmacological treatments of substance use disorders [2]. A common cause of craving is exposure to drug-related conditioned cues, but patients often report that strong feelings of craving "just came over me" for no apparent reason. An intriguing hypoth- 
esis is that such quick, emotional responses can be elicited by cues that are so subtle that they are only perceived subliminally, i.e., without conscious awareness [3].

Subliminal visual processing has been studied with different methods, one of them being backward visual masking. In this paradigm, a very brief visual stimulus (the target) is immediately followed by another picture (the mask), which disturbs the perception of the target stimulus so that it does not reach conscious awareness [4]. This paradigm has been important in studies of fear, where the amygdala has been shown to play a central role in a subcortical network that is able to detect fear-relevant stimuli and elicit fear responses in a fast and nonconscious manner [5].

Many studies have explored subliminal processes in the fear and avoidance system and found them to have clinical relevance, but there are also studies of appetitive or positive motivational systems, not least in substance use disorders. Early behavioral studies of smokers using masked Stroop tasks and other tests to look for attentional biases toward smoking-related stimuli did not find any evidence for subliminal cue reactivity [6-8], although one study found that a subgroup of smokers that had been deprived of cigarettes for $12 \mathrm{~h}$ exhibited a processing bias for subliminally presented smoking stimuli [9]. More recently, the masking paradigm has been employed in fMRI studies of substance-dependent patients. A first pilot study of cocaine-dependent patients reported activations in several limbic structures when the patients were exposed to masked visual cocaine cues, suggesting that cue reactivity could be evoked by subliminal stimuli in persons with substance use disorders, indicating a heightened neural sensitivity similar to persons with, e.g., phobias [3]. In 2014, the same research group published a study suggesting that the $\mathrm{GABA}_{\mathrm{B}}$ receptor agonist baclofen could block this subliminal cue reactivity [10]. In another study, cannabis-dependent patients were reported to have similar levels of subliminal cue reactivity as the cocaine-dependent patients [11].

In 2009, subliminal cue reactivity was investigated in a small study of nicotine-dependent patients [12]. In that particular study, the right amygdala showed a decreased BOLD signal in response to masked smoking-related images, a result which is opposite to that of previous studies in cocaine patients [3]. Limited sample sizes and differences in patient populations, task designs, and durations of cue exposure might all have contributed to the heterogeneity of these results.

In summary, the fMRI studies published so far on subliminal drug cue reactivity in substance use disorders have all reported statistically significant results, but the sample sizes have been small and the results not entirely consistent. We therefore decided to investigate this as part of an fMRI project on cue reactivity in amphetamine dependence, where we would have access to a larger sample of amphetamine-dependent subjects and healthy controls. The larger project also aimed to investigate the impact of the opioid antagonist naltrexone on cue reactivity and craving [13], since naltrexone has earlier been shown to attenuate subjective effects of amphetamine such as arousal and craving $[14,15]$, and it possibly also reduces the risk of relapse in amphetamine-dependent patients [16]. In this experiment, our hypothesis was that subliminally presented drug cues, compared to neutral cues, would activate the amygdala and striatum in amphetamine-dependent subjects but not in healthy controls. A secondary hypothesis was that the subliminal cue reactivity would be attenuated in naltrexone-treated subjects, compared to placebo.

\section{Materials and Methods}

This experiment was part of a larger study designed as a randomized, double-blind, placebo-controlled clinical trial with parallel groups, including both amphetamine dependent subjects and healthy controls. Each subject received one oral dose of naltrexone (50 mg) or placebo and subsequently went through an fMRI examination, investigating drug cue reactivity and craving. The study was approved by the Stockholm Ethics Review Board and the Swedish Medical Products Agency and preregistered in the EU Clinical Trials Register (EudraCT 2010-021384-33). The study was performed according to CONSORT guidelines and International Conference on Harmonisation guidelines for Good Clinical Practice, with external monitoring by the Karolinska Trial Alliance. All participants provided their written, informed consent before any study procedures took place.

\section{Participants}

Forty male, non-treatment-seeking amphetamine users aged 20-65 were recruited via advertisement and word of mouth at the needle exchange program and in shelters in the Stockholm region. Thirty male, healthy control subjects were recruited via word of mouth and advertisement at Karolinska Institutet.

All participants were screened by a study physician. For amphetamine users, this included psychiatric assessment with the Structured Clinical Interview for DSM-IV, Axis 1 (SCID-1), and detailed assessment of substance use history and other background variables with the Addiction Severity Index [17]. Inclusion criteria included DSM-IV diagnosis of amphetamine dependence since at least 2 years, history of intravenous amphetamine use, amphetamine use for minimum of 12 days in the last 12 weeks, and having been drug free for 1-30 days (minimum $24 \mathrm{~h}$ ). Exclusion criteria were other current substance dependence (except nicotine), schizophrenia or bipolar disorder I, left-handedness, clinical signs of amphetamine intoxication at the day of testing, traces of can- 
Fig. 1. Subliminal cue presentation paradigm, with active cue and mask, followed by a fixation cross before the onset of the next cue. In trials with a delayed mask, a black screen was shown for $94 \mathrm{~ms}$ between the cue and the mask.

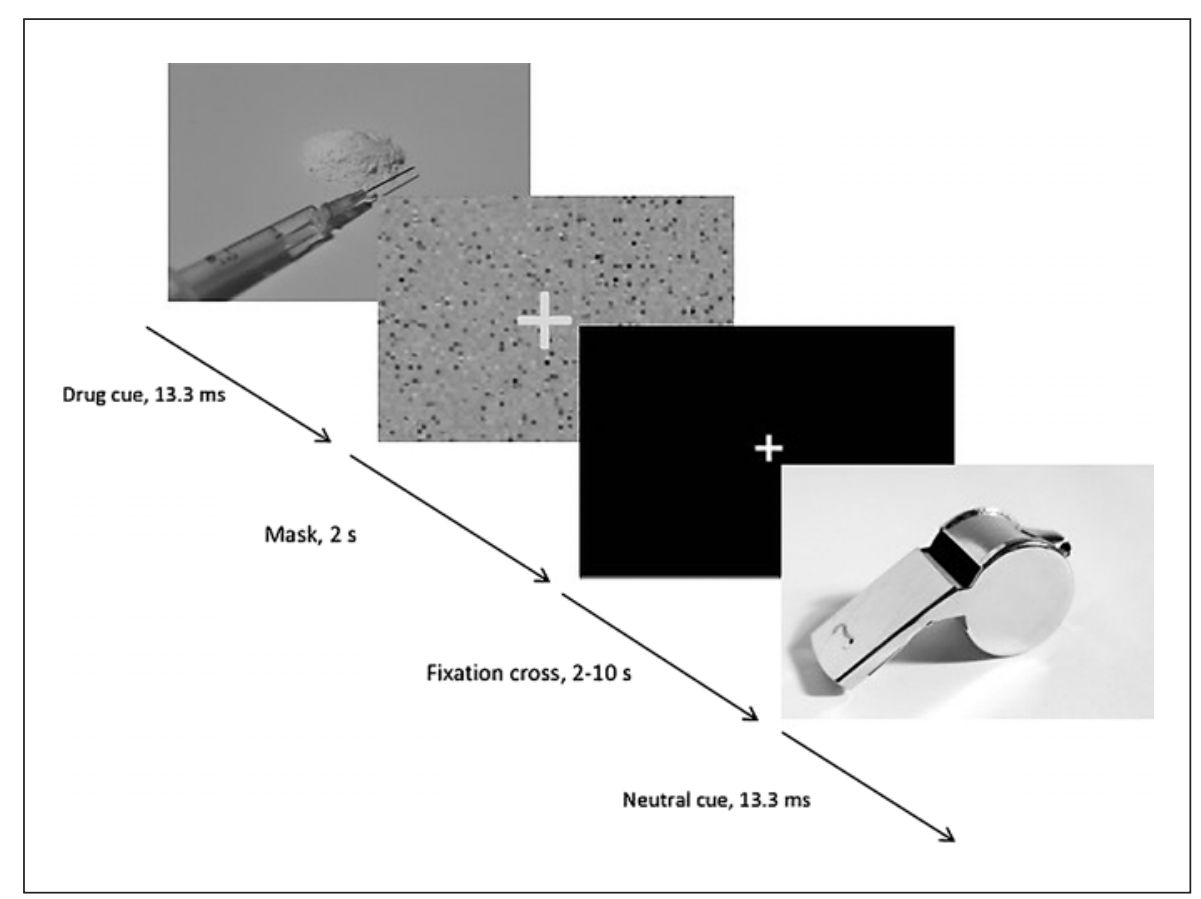

nabis, opiates, cocaine, or benzodiazepines in the urine at the day of testing, traces of alcohol as measured by using a breathalyzer at the day of testing, or presence of severe somatic disease.

For control subjects, psychiatric screening was done with the MINI interview [18], and substance use was assessed with the AUDIT [19] and DUDIT [20] screening tools. Exclusion criteria were DSM-IV diagnosis of any substance-dependence disorder in self or in first-degree relatives, DSM-IV diagnosis of any major psychiatric illness, left-handedness, traces of drugs in the urine at test day, traces of alcohol as measured with a breathalyzer on the test day, and use of any concomitant medication. Subjects with contraindications to MRI (e.g., cardiac pacemaker and severe claustrophobia) or the study medication (e.g., regular opioid use) were also excluded.

\section{Study Procedures}

After a first screening visit, eligible subjects were scheduled for a test day. Having arrived in the clinic on the test day, they were asked for a supervised urine test to exclude current use of drugs. If the result was negative and a breathalyzer test showed no trace of alcohol, the subject was included and randomized to receive a capsule of $50 \mathrm{mg}$ naltrexone or placebo. After an interval of at least 60 $\mathrm{min}$, the fMRI procedures were started.

The experiment described here consisted of the subjects lying in a supine position in the MR scanner and being presented with amphetamine-related (i.e., active) or neutral cue pictures using Presentation software (Neurobehavioral Systems, Inc.) and visual goggles (binocular OLED display, resolution $800 \times 600$, and frequency $75 \mathrm{~Hz}$; Nordic Neuro Lab, Bergen, Norway). Subjects were given the following instruction: "In this experiment, you will be exposed to a number of pictures that are shown very quickly. You might be able to distinguish some of them, but many of them will be hard to see at all. Your task is to lie still and pay full attention to what is happening on the screen." The active cues consisted of pictures of amphetamine and paraphernalia such as syringes, while the neutral showed everyday items such as a dishcloth, a whistle, and a glass of water (see Fig. 1 for example). Each target picture was shown for $13.3 \mathrm{~ms}$, followed by a mask picture that was presented either immediately or after a delay of $94 \mathrm{~ms}$, with only a black screen shown during the delay. This was done to assess whether a delayed onset of the mask, presumably attenuating the masking effect, would affect the processing of the target stimulus.

The mask was constructed by scrambling the target picture and adding a gray fixation cross, in order to keep global color and luminance constant while still disturbing the processing of the target. The short target stimulus duration (13.3 ms) was chosen empirically based on pilot data obtained outside the MR scanner, with the exact same paradigm and visual goggles used in the imaging experiment. We found that when the duration of the cue stimuli was prolonged beyond $13.3 \mathrm{~ms}$, healthy subjects $(n=7)$ could discriminate between drug and neutral cues better than chance in an immediate forced choice paradigm, which is the standard definition of the threshold for conscious access of information [4]. With a cue duration of $13.3 \mathrm{~ms}$ however, they could not discriminate better than chance, and we therefore settled for this cue duration to ensure that the cues would not be consciously perceived. Although this cue duration is shorter than in previous studies of subliminal cue reactivity in substance use disorders, it is similar to that of many other studies of subliminal perception [21-23].

The order of the presented stimuli was randomized, and each target cue + mask was separated by a fixation cross for a variable interval of $2-10 \mathrm{~s}$ (Fig. 1). There were 20 masked target pictures in each of the 4 categories: active with mask, neutral with mask, active with delayed mask, and neutral with delayed mask. The experiment lasted for a total of $7 \mathrm{~min}$ (242 image volumes acquired). 


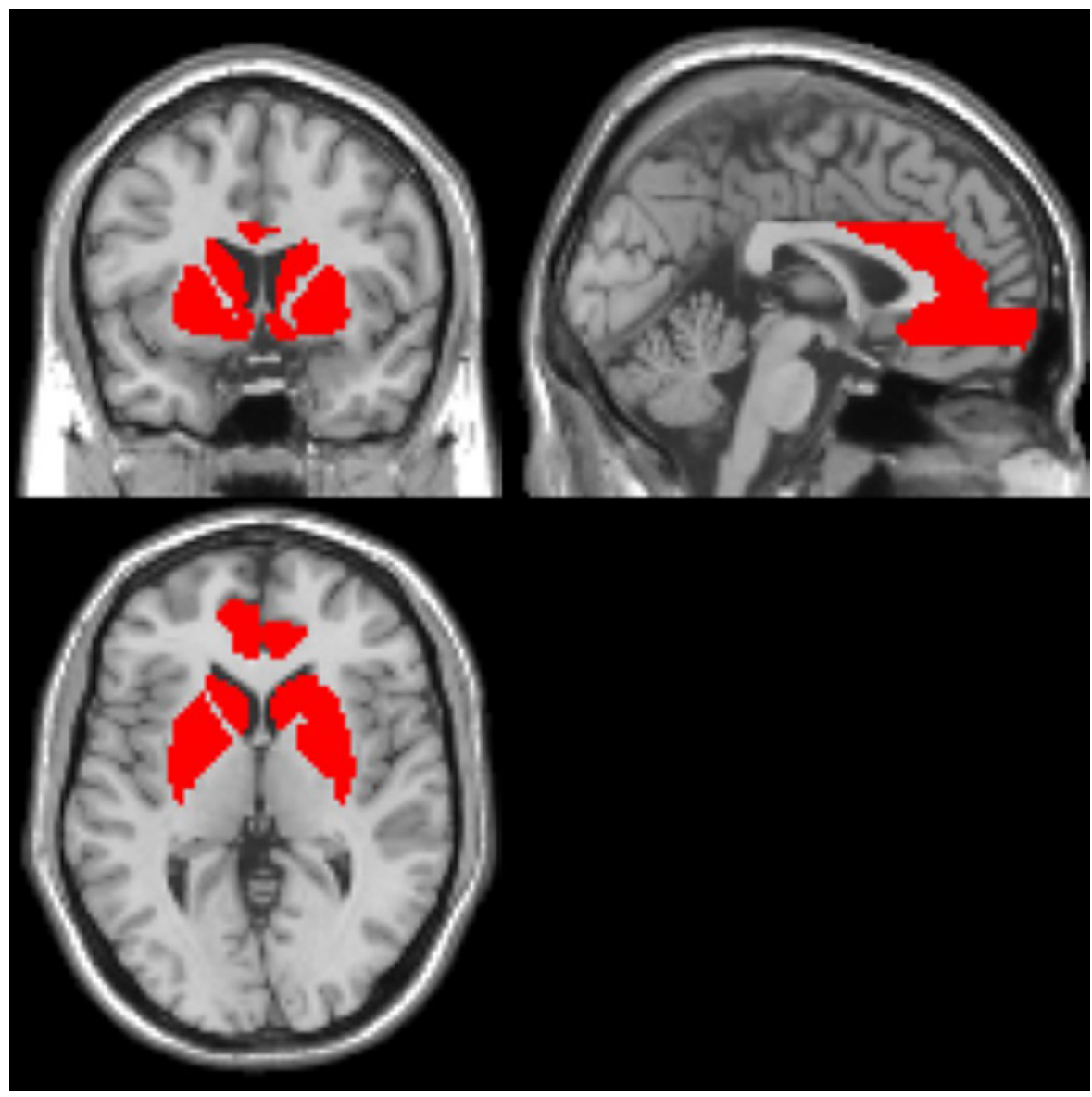

Fig. 2. Brain mask used in the primary analysis, constructed with the WFU PickAtlas software. The included regions are marked in red: the amygdala, ventral tegmental area, striatum, anterior cingulate cortex, orbitofrontal cortex, and medial prefrontal cortex.

\section{Magnetic Resonance Imaging}

MRI examinations were performed with a $3 \mathrm{~T}$ instrument (GE MR750 Discovery) with an 8-channel head coil at the Karolinska MR Research Center. Each subject went through a total of $4 \mathrm{fMRI}$ runs as well as structural imaging. The MR scanning session lasted for about $50 \mathrm{~min}$ in total. The fMRI run described here was the first task-related fMRI run that was performed in the imaging session, preceded only by fMRI resting state data acquisition. T2*-sensitized BOLD fMRI images were acquired using a gradient-echo echo-planar imaging sequence (slice thickness $=2.3 \mathrm{~mm}$, number of slices $=36, \mathrm{TR}=2114 \mathrm{~ms}, \mathrm{TE}=30 \mathrm{~ms}$, flip angle $=75^{\circ}$, and FOV $=280 \mathrm{~mm}$ ). In order to maximize the spatial resolution in our regions of interest in the limbic system, some dorsal parts of the cerebral cortex (e.g., primary sensory and motor areas) were not covered by the functional sequences in most subjects.

\section{Data Analysis}

fMRI data were analyzed within the Statistical Parametric Mapping software platform, SPM 12 [24]. Functional MRI images were realigned, co-registered to the participant's structural image, segmented, and normalized to the MNI brain atlas as implemented in the SPM software. Finally, all fMRI images were spatially smoothed using a Gaussian kernel with a full-width-half-maximum of $7 \mathrm{~mm}$. Confounding effects from subject head movement during data acquisition are a critical problem in fMRI, particularly when com- parisons between clinical and healthy cohorts are made [25-27]. We anticipated that amphetamine users might exhibit more head movement than healthy, drug-naïve subjects, and this potential source of bias warranted particular care in the analysis.

To this end, we performed a quantitative screening of realignment image movement using the frame-wise displacement (FD) parameter [25]. An estimation of the FD is computed for each frame (image volume) as the root-mean-squared difference between adjacent images in terms of rotational and translational movements (in total 6 movement parameters). Next, we computed the mean FD value (taken over all image volumes) for each subject and performed a 2-sample $t$ test to test for differences in mean FD values between groups. We observed a significantly larger mean FD value in the patient cohort compared to the control cohort $(p=0.028, T=2.26)$, which suggests that the fMRI data collected in amphetamine users were indeed significantly more influenced by head movement than controls. This result led us to be cautious in terms of which fMRI data to include in the final analysis. Hence, we employed an a priori subject-level threshold where we only included subjects for which less than $25 \%$ of the image volume (frames) had an FD value larger than 0.3 (see also [25]).

The functional data were analyzed in an event-related design using the general linear model, where the primary contrast was active with immediate mask $>$ neutral with immediate mask, and 
Table 1. Demographics and drug use history

\begin{tabular}{lllr}
\hline & $\begin{array}{l}\text { Amphetamine } \\
\text { users }\end{array}$ & $\begin{array}{l}\text { Healthy } \\
\text { participants }\end{array}$ & $p$ value \\
\hline Age & $44.1 \pm 9.9$ & $40.3 \pm 8.3$ & 0.084 \\
Years of amphetamine use & $18.7 \pm 9.8$ & 0 & $<0.001$ \\
Days of amphetamine use during last month & $22.0 \pm 10.2$ & 0 & $<0.001$ \\
Days since last amphetamine use & $4.7 \pm 4.5$ & $\mathrm{~N} . \mathrm{A}$ & $\mathrm{NA}$ \\
Chronic viral hepatitis & $65 \%$ & $0 \%$ & $<0.001$ \\
HIV & $5 \%$ & $0 \%$ & 0.214 \\
Nicotine dependence & $85 \%$ & $0 \%$ & $<0.001$ \\
Living alone & $85 \%$ & $40 \%$ & $<0.001$ \\
Years in school & $10.7 \pm 3.1$ & $16.2 \pm 2.5$ & $<0.001$ \\
Months incarcerated & $53.5 \pm 91.2$ & 0 & 0.001 \\
\hline
\end{tabular}

Means, standard deviations, and $p$ values from Welch's $t$ tests are provided for continuous variables and $p$ values from $x^{2}$ tests for categorical variables. movement parameters and FD were included as covariates of no interest. All results were corrected for multiple comparisons using family-wise error correction with level of significance set at $p<$ 0.05 . For the primary analysis, we used the WFU PickAtlas software (http://fmri.wfubmc.edu/software/pickatlas) to assemble a mask in order to limit the parameter space to prespecified regions of interest, based on the prior literature on subliminal perception and drug cue reactivity [28]. The regions included in the image mask were the amygdala, ventral tegmental area, striatum, anterior cingulate cortex, orbitofrontal cortex, and medial prefrontal cortex, regions that all have been implicated in earlier studies of conscious cue reactivity in stimulant users (Fig. 2) [28, 29]. We also did a whole-brain analysis to investigate possible subliminal cue reactivity in other brain regions (although it should be noted that some dorsal parts of the cortex were not always included in the functional sequences, as mentioned above). The amphetamine users and healthy controls were analyzed separately. We did not differentiate between naltrexone- and placebo-treated subjects in the primary analysis, but in a secondary analysis, we compared the effect of naltrexone versus placebo.

\section{Results}

The amphetamine users recruited were $44 \pm 10$ years old and exhibited long histories of severe amphetamine dependence, with a mean of 19 years of amphetamine use and having used amphetamine on average 22 days in the latest month. Further information on the demographics and drug use history is provided in Table 1, and psychiatric history is provided in online supplementary document 1 (for all online suppl. material, see www. karger.com/doi/10.1159/000521344). The healthy participants were of a similar age, but differed on variables such as nicotine dependence and social and educational background. None of the groups had any significant dif- ferences in baseline variables between the naltrexoneand placebo-treated participants.

Forty amphetamine users were randomized, but 10 of them were unable to successfully complete the fMRI examination because of suspected adverse reactions to the medication $(n=4)$, claustrophobia $(n=2)$, or technical problems $(n=4)$. In the group of healthy subjects, 4 persons could not perform the examination because of technical problems and one interrupted the examination because of claustrophobia. In total, 30 amphetamine users and 25 controls contributed data. Head movements were common in the amphetamine group, and our strict threshold for head movements meant that fMRI data from 6 subjects were discarded before data were analyzed, leaving 24 amphetamine users (12 in the naltrexone- and 12 in the placebo-treated group) and 25 healthy subjects (13 in the naltrexone- and 12 in the placebo-treated group) with complete data and no major movement artifacts. In our primary contrast of active with immediate mask $>$ neutral with immediate mask, the patient group exhibited no statistically significant activations in our predefined regions of interest.

We did not find any significant activations when extending the analysis to the whole brain, although it should again be noted that some of the most dorsal parts of the cerebral cortex were not covered in most subjects. The same results were found in the healthy control group, and we found no effect of naltrexone versus placebo in any of the groups, although the sample size may have been too small to reach adequate power for such a comparison. The small clusters found with an uncorrected threshold are described in Table 2 . The secondary analysis of active with delayed mask $>$ neutral with delayed mask did not 
Table 2. SPM results for the main contrasts (subliminal = with immediate mask, supraliminal = with delayed mask)

\begin{tabular}{|c|c|c|c|c|c|}
\hline $\begin{array}{l}\text { Subliminal drug cue }>\text { neutral cue } \\
\text { (amphetamine users) }\end{array}$ & $\begin{array}{l}-28,-6,-2 \text { (left putamen) } \\
-30,0,-26 \text { (left amygdala) } \\
22,22,-8 \text { (right putamen) } \\
12,58,-14 \text { (right orbitofrontal cortex) } \\
22,26,10 \text { (right caudate) }\end{array}$ & 1.000 & $\begin{array}{l}3.28 \\
3.21 \\
2.93 \\
2.92 \\
2.91\end{array}$ & $\begin{array}{l}2.94 \\
2.89 \\
2.68 \\
2.66 \\
2.66\end{array}$ & $\begin{array}{l}19 \\
2 \\
1 \\
1 \\
1\end{array}$ \\
\hline $\begin{array}{l}\text { Subliminal drug cue }>\text { neutral cue } \\
\text { (healthy controls) }\end{array}$ & $32,0,12$ (right putamen) & 1.000 & 3.08 & 2.80 & 2 \\
\hline $\begin{array}{l}\text { Naltrexone > placebo } \\
\text { (amphetamine users) }\end{array}$ & $\begin{array}{l}-18,12,-6 \text { (left putamen) } \\
-20,2,12 \text { (left putamen) }\end{array}$ & 1.000 & $\begin{array}{l}3.71 \\
3.35\end{array}$ & $\begin{array}{l}3.18 \\
2.93\end{array}$ & $\begin{array}{l}24 \\
2\end{array}$ \\
\hline $\begin{array}{l}\text { Supraliminal drug cue }>\text { neutral cue } \\
\text { (healthy controls) }\end{array}$ & $\begin{array}{l}46,28,22 \text { (right inferior frontal gyrus) } \\
-62,-38,30 \text { (left supramarginal gyrus) } \\
-50,-20,22 \text { (left rolandic operculum) } \\
-38,-6,2 \text { (left insula) } \\
30,60,-6 \text { (right orbitofrontal cortex) }\end{array}$ & $\begin{array}{l}0.925 \\
0.962 \\
0.993 \\
0.998 \\
0.999\end{array}$ & $\begin{array}{l}4.06 \\
3.94 \\
3.70 \\
3.56 \\
3.48\end{array}$ & $\begin{array}{l}3.51 \\
3.43 \\
3.26 \\
3.16 \\
3.10\end{array}$ & $\begin{array}{l}23 \\
8 \\
3 \\
2 \\
1\end{array}$ \\
\hline
\end{tabular}

Note that none of the activations were statistically significant after correction. The Naltrexone $>$ placebo contrast for healthy controls is not shown, since no significant voxels were found even with an uncorrected threshold of $p<0.005$.

reveal any statistically significant activations either (see also Table 2). Further, exploratory analyses are provided in online supplementary document 1 . In short, these analyses indicated that amphetamine users might show increased activation of the left hippocampus in response to drug versus neutral cues and enhanced activation in visual areas, compared to healthy controls, as indicated in online supplementary Figures 1a and 2c. However, these findings need to be interpreted with caution, as these analyses do not adhere to current recommendations for the use of cluster thresholding in functional neuroimaging data analysis [30].

\section{Discussion}

In this sample of amphetamine users and healthy subjects, we found no evidence of specific neural reactivity to subliminally presented drug cues. To our knowledge, this is the largest imaging study so far investigating the hypothesis of subliminal cue reactivity in substance use disorders, and our results are consistent with most of the behavioral studies of nicotine dependence described earlier [6-8]. However, as the results are in contrast with the conclusions of the few earlier published imaging studies, a closer look at methodological differences between our study and previous findings is warranted.

In the present study, we selected a subliminal cue duration of $13.3 \mathrm{~ms}$ based on pilot data, as described above. Other studies of subliminal cue reactivity in substance use disorders have settled for somewhat longer cue durations, which might in part be explained by differences between the different kinds of equipment used for stimulus presentation [30]. We used goggles with OLED displays, which have a slower picture decay than the LCD projectors used in the previously published studies. This means that the actual time duration of the target cue at the level of the retina might be somewhat longer than $13.3 \mathrm{~ms}$. Reactions to subliminal stimuli presented with time durations similar to ours have been described in the literature [21-23], suggesting that the chosen parameters are appropriate for this technique.

There are also some differences in the preprocessing pipeline and analysis of neuroimaging data between the 
present study and previous work. One point to consider is movement artifacts, which can be problematic in patient populations that may have problems lying still for long periods of time $[31,32]$. Although we carefully instructed the subjects in our study to lie still and used foam pads to restrict head movements, we still had to exclude 6/30 amphetamine users because of excessive movement artifacts. However, none of the previously published studies on this topic report any thresholds for movement artifacts or whether any data were lost because of this.

Another methodological question is the way that statistical inference is carried out on the fMRI data. In the 2008 pilot study of cocaine-dependent patients, there was no correction for multiple comparisons, which entails an increased risk of false-positive findings [3]. In a more recent study from the same group, baclofen was suggested to block the subliminal cue reactivity [10], but the sample size was small ( $n=10$ per group), and data from 7 of the placebo-treated patients had already been included in the earlier pilot study. In a third study, data from 20 cannabis-dependent patients were interpreted as evidence of subliminal cue reactivity similar to the one in cocaine patients [11]. However, in this as well as the baclofen study, the methods for controlling the type I error rate could have been stricter. While regular SPM methods were used for preprocessing and first-level analyses, the group-level statistical analysis used 3dClustSim of the AFNI software package, a method that unfortunately has been shown not only to underestimate group smoothness but also to contain a bug (corrected as of May 2015), which together significantly increased the risk of false positives [33]. Therefore, the results of these studies must be interpreted with caution. Also, none of these studies included any healthy control group, which would be needed to validate the hypothesis of a subliminal drug cue reactivity specific to subjects with substance use disorders.

As mentioned in the Introduction section, another research group published a study of subliminal cue reactivity in cigarette smokers in 2009, which actually suggested a deactivation of the amygdala, i.e., an effect in the opposite direction compared to that of the 2008 pilot study on cocaine users [12]. However, the small sample size $(n=7)$ makes this finding less certain. Whether there are other, still unpublished attempts at replication of these findings is unclear. Recent meta-analyses have indicated that negative results are underreported in cognitive neuroscience [34-37].

It is worth noting that this study was conducted with amphetamine-dependent subjects, while the earlier have included cocaine, cannabis, and nicotine users. However, it is hard to see any clear reasons why subliminal cue reactivity would differ very much between amphetamine and cocaine users, for instance. This sample of amphetamine users also exhibited strong neural and behavioral reactivity to supraliminal drug cues, as reported earlier [13].

In contrast to the case of subliminal fear reactivity, there is not much evidence from other sources regarding subliminal reactivity for drug-related stimuli in substance use disorders. While very fast and somewhat unspecific fear responses to threatening visual stimuli have obvious advantages from an evolutionary point of view for avoiding predators, the fitness value of similarly fast appetitive responses is less clear, since unspecific reactions come at a metabolic cost. The clinical observation that patients often cannot describe the cause of their craving might be explained by other mechanisms, such as the increasingly habitual nature of drug-seeking behavior in the course of addiction [38]. Research on fear in both humans and animals has also delineated a subcortical pathway for such responses, the so-called low road from the retina via the superior colliculus and pulvinar nucleus of the thalamus to the amygdala, but such models have not yet been developed and tested for appetitive stimuli such as drug cues for addicted patients [5, 39]. Some recent fMRI studies have reported neural reactivity to subliminal sexual cues in healthy volunteers, which might be a suitable paradigm to explore further $[40,41]$. Instead of passive cue exposure, future studies of addicted subjects could also be inspired by more sophisticated paradigms and learning tasks that have been employed in studies of subliminal learning in healthy participants [42]. This might also allow for more direct behavioral validation of the experimental procedures, in order to put any neuroimaging findings in context. One could also consider tasks related to emotional regulation, since this is often impaired in patients with substance use disorders, and altered reactivity to subliminal emotional stimuli might be a relevant mechanism behind these impairments [43].

\section{Limitations}

Although this is the largest neuroimaging study published so far investigating subliminal drug cue reactivity, the sample size and resulting statistical power are still limited, considering the supposedly weak effects of subliminal cues on the BOLD signal. We also had to exclude several subjects due to excessive movements during data acquisition, thereby reducing the sample size but in- 
creasing the precision of the measurements. The fact that half of the participants in this study were pretreated with naltrexone could hypothetically have made them less reactive to the cues, thereby reducing the overall signal in analyses including both treatment groups. However, we found no differences between naltrexone- and placebo-treated subjects in the data, and it is unclear whether naltrexone affects the processing even of consciously perceived drug cues in stimulant-dependent patients $[13,44]$. Another issue is our use of a shorter cue duration, compared to earlier studies. This choice was based on pilot data, to make sure that the stimuli were indeed presented subliminally: with longer cue durations, many participants could discriminate between drug and neutral cues better than chance. However, the participants in these pilot sessions were healthy and drug-free, and it is possible that the threshold of conscious perception could be higher in subjects with long histories of intravenous drug use, such as the patient group in this study. The somewhat surprising fact that we did not see any significant BOLD activations when comparing active and neutral cues with delayed masks might indicate that this could be the case. However, there is to our knowledge no published systematic investigations of perceptual thresholds to consciousness in patients with substance use disorders compared to controls. The lack of significant activations in the delayed mask condition is most likely due to limited visibility: although we do not have validation data on the ability of this specific picture set to elicit cue reactivity, clearly visible pictures of drugs and paraphernalia have been shown to activate motivationally relevant neurocircuitry in stimulant users in a number of earlier studies, including another experiment from this study, where we found highly significant neural cue reactivity from visual stimuli of similar content $[13,44]$. The use of differing techniques for the display of stimuli might also have affected the stimuli durations: future studies should aim to use similar equipment in order to facilitate comparisons between studies. Another limitation is that we did not perform a forced choice recognition task following this experiment, to make sure that the subjects did not consciously perceive the masked images. However, given the short stimulus duration and the lack of neural reactivity, this seems unlikely. Last, this experiment only included male participants because of features in other parts of the study not presented here. Since gender might influence the neural patterns of drug cue reactivity in substance use disorders, we cannot tell if the inclusion of female subjects would have yielded other results [45].

\section{Conclusions}

In summary, we did not find any evidence that subliminally presented visual drug cues activate motivationally relevant neurocircuitry in the brains of amphetamine-dependent subjects. On closer reading of the earlier literature, we found methodological concerns that make previous claims of subliminal cue reactivity in addiction less certain. Further studies are therefore needed to clarify if such reactivity can be demonstrated with rigorous methods in larger samples. Other experimental paradigms may also be needed to investigate the potential role of unconscious processes in substance use disorders.

\section{Acknowledgments}

We thank Camilla Hellspong and the staff at the Magnus Huss clinic for assistance with the clinical procedures of the study and the Stockholm Needle Exchange and Stockholm Drug Users Union for helping to recruit study participants.

\section{Statement of Ethics}

This study was approved by the Stockholm Ethics Review Board (Nr 2010/930-31/1), and all participants provided their written, informed consent before any study procedures took place.

\section{Conflict of Interest Statement}

J.F. is an editorial board member of European Addiction Research. The authors have no conflicts of interest to declare.

\section{Funding Sources}

This work was funded by grants from the Stockholm County Council (Forskar-ST; a "research residency" grant), Svenska Sällskapet för Medicinsk Forskning (a postdoctoral research grant), Märta Lundqvist Foundation and Professor Bror Gadelius Memorial Fund to Joar Guterstam, the Swedish Research Council (201361X-21444-04-3), the Swedish Research Council for Health, Working life and Welfare (2013-1849), and the Swedish Brain Foundation to Johan Franck, and the Swedish Research Council to Nitya Jayaram-Lindström (2009-34201-69804-23). The funding sources had no role in the design or procedures of the study, nor in the interpretation of the data, the writing of the report, or the decision to submit it for publication. 


\section{Author Contributions}

J.G., N.J.L., P.P., M.I., and J.F. conceived the study. J.G. and J.B. contributed to data collection. P.F., J.B., J.G., and P.P. contributed to data analysis and interpretation. J.G. wrote the initial manuscript draft. All authors edited and approved the final manuscript.

\section{Data Availability Statement}

The data generated in this study are not publicly available, since this was not included in the informed consent signed by the participants. Questions about access to data can be made to the corresponding author.

\section{References}

1 Tiffany ST, Wray JM. The clinical significance of drug craving. Ann N Y Acad Sci. 2012 Feb; 1248:1-17.

2 O'Brien CP. Anticraving medications for relapse prevention: a possible new class of psychoactive medications. Am J Psychiatry. 2005 Aug;162(8):1423-31.

3 Childress AR, Ehrman RN, Wang Z, Li Y, Sciortino N, Hakun J, et al. Prelude to passion: limbic activation by "unseen" drug and sexual cues. PLoS One. 2008 Jan 30;3(1):e1506.

4 Kouider S, Dehaene S. Levels of processing during non-conscious perception: a critical review of visual masking. Philos Trans R Soc Lond B Biol Sci. 2007 May 29;362(1481):857-75.

5 Ohman A, Carlsson K, Lundqvist D, Ingvar $\mathrm{M}$. On the unconscious subcortical origin of human fear. Physiol Behav. 2007 Sep 10;92(12):180-5.

6 Mogg K, Bradley BP. Selective processing of smoking-related cues in smokers: manipulation of deprivation level and comparison of three measures of processing bias. J Psychopharmacol. 2002 Dec;16(4):385-92.

7 Munafò M, Mogg K, Roberts S, Bradley BP, Murphy M. Selective processing of smokingrelated cues in current smokers, ex-smokers and never-smokers on the modified Stroop task. J Psychopharmacol. 2003 Sep;17(3): 310-6.

8 Bradley B, Field M, Mogg K, De Houwer J. Attentional and evaluative biases for smoking cues in nicotine dependence: component processes of biases in visual orienting. Behav Pharmacol. 2004 Feb;15(1):29-36.

9 Leventhal AM, Waters AJ, Breitmeyer BG, Miller EK, Tapia E, Li Y. Subliminal processing of smoking-related and affective stimuli in tobacco addiction. Exp Clin Psychopharmacol. 2008 Aug;16(4):301-12.

10 Young KA, Franklin TR, Roberts DCS, Jagannathan K, Suh JJ, Wetherill RR, et al. Nipping cue reactivity in the bud: baclofen prevents limbic activation elicited by subliminal drug cues. J Neurosci. 2014 Apr 2;34(14):5038-43.

11 Wetherill RR, Childress AR, Jagannathan K, Bender J, Young KA, Suh JJ, et al. Neural responses to subliminally presented cannabis and other emotionally evocative cues in cannabis-dependent individuals. Psychopharmacology. 2014 Apr;231(7):1397-407.

12 Zhang X, Chen X, Yu Y, Sun D, Ma N, He S, et al. Masked smoking-related images modulate brain activity in smokers. Hum Brain Mapp. 2009 Mar;30(3):896-907.
13 Guterstam J, Jayaram-Lindstrom N, Berrebi J, Petrovic $\mathrm{P}$, Ingvar M, Fransson $\mathrm{P}$, et al. Cue reactivity and opioid blockade in amphetamine dependence: a randomized, controlled fMRI study. Drug Alcohol Depend. 2018 Jan; 191:91-7.

14 Jayaram-Lindström N, Konstenius M, Eksborg S, Beck O, Hammarberg A, Franck J. Naltrexone attenuates the subjective effects of amphetamine in patients with amphetamine dependence. Neuropsychopharmacology. 2008;33(8):1856-63.

15 Jayaram-Lindstrom N, Guterstam J, Haggkvist J, Ericson M, Malmlof T, Schilstrom B, et al. Naltrexone modulates dopamine release following chronic, but not acute amphetamine administration: a translational study. Transl Psychiatry. 2017 Apr 25;7(4):e1104.

16 Jayaram-Lindström N, Hammarberg A, Beck $O$, Franck J. Naltrexone for the treatment of amphetamine dependence: a randomized, placebo-controlled trial. Am J Psychiatry. 2008;165(11):1442-8.

17 McLellan AT, Kushner H, Metzger D, Peters R, Smith I, Grissom G, et al. The Fifth Edition of the Addiction Severity Index. J Subst Abuse Treat. 1992;9(3):199-213.

18 Sheehan DV, Lecrubier Y, Sheehan $\mathrm{KH}$, Amorim P, Janavs J, Weiller E, et al. The miniinternational neuropsychiatric interview (M.I.N.I.): the development and validation of a structured diagnostic psychiatric interview for DSM-IV and ICD-10. J Clin Psychiatry. 1998;59(Suppl 20):22-33; quiz 34-57.

19 Babor T, Higgins-Biddle J, Saunders J, Monteiro M. AUDIT. The Alcohol Use Disorders Identification Test. Guidelines for use in primary care. 2nd ed. WHO; 2001.

20 Berman AH, Bergman H, Palmstierna T, Schlyter F. Evaluation of the drug use disorders identification test (DUDIT) in criminal justice and detoxification settings and in a Swedish population sample. Eur Addict Res. 2005;11(1):22-31.

21 Pegna AJ, Darque A, Berrut C, Khateb A. Early ERP modulation for task-irrelevant subliminal faces. Front Psychol. 2011;2:88.

22 Reber TP, Henke K. Rapid formation and flexible expression of memories of subliminal word pairs. Front Psychol. 2011;2:343.

23 Züst MA, Colella P, Reber TP, Vuilleumier P, Hauf M, Ruch S, et al. Hippocampus is place of interaction between unconscious and conscious memories. PLoS ONE. 2015;10(3): e0122459.
24 Friston KJ, Holmes AP, Worsley KJ, Poline J-P, Frith CD, Frackowiak RSJ. Statistical parametric maps in functional imaging: a general linear approach. Hum Brain Mapp. 1994;2(4):189-210.

25 Power JD, Barnes KA, Snyder AZ, Schlaggar BL, Petersen SE. Spurious but systematic correlations in functional connectivity MRI networks arise from subject motion. Neuroimage. 2012 Feb 1;59(3):2142-54.

26 Satterthwaite TD, Wolf DH, Loughead J, Ruparel K, Elliott MA, Hakonarson $\mathrm{H}$, et al. Impact of in-scanner head motion on multiple measures of functional connectivity: relevance for studies of neurodevelopment in youth. Neuroimage. 2012 Mar;60(1):623-32.

27 Van Dijk KR, Sabuncu MR, Buckner RL. The influence of head motion on intrinsic functional connectivity MRI. Neuroimage. 2012 Jan 2;59(1):431-8.

28 Chase HW, Eickhoff SB, Laird AR, Hogarth L. The neural basis of drug stimulus processing and craving: an activation likelihood estimation meta-analysis. Biol Psychiatry. $2011 \mathrm{Oct}$ 15;70(8):785-93.

29 Jasinska AJ, Stein EA, Kaiser J, Naumer MJ, Yalachkov Y. Factors modulating neural reactivity to drug cues in addiction: a survey of human neuroimaging studies. Neurosci Biobehav Rev. 2014 Jan;38:1-16.

30 Eklund A, Nichols TE, Knutsson H. Cluster failure: why fMRI inferences for spatial extent have inflated false-positive rates. Proc Natl Acad Sci U S A. 2016 12;113(28):7900-5.

31 Wiens S, Fransson P, Dietrich T, Lohmann P, Ingvar M, Ohman A. Keeping it short: a comparison of methods for brief picture presentation. Psychol Sci. 2004 Apr;15(4):282-5.

32 Friston KJ, Williams S, Howard R, Frackowiak RS, Turner R. Movement-related effects in fMRI time-series. Magn Reson Med. 1996 Mar;35(3):346-55.

33 Zeng L-L, Wang D, Fox MD, Sabuncu M, Hu D, Ge M, et al. Neurobiological basis of head motion in brain imaging. Proc Natl Acad Sci USA. 2014 Apr 22;111(16):6058-62.

34 Carp J. The secret lives of experiments: methods reporting in the fMRI literature. Neuroimage. 2012 Oct 15;63(1):289-300.

35 Button KS, Ioannidis JPA, Mokrysz C, Nosek BA, Flint J, Robinson ESJ, et al. Power failure: why small sample size undermines the reliability of neuroscience. Nat Rev Neurosci. 2013;14(5):365-76. 
36 David SP, Ware JJ, Chu IM, Loftus PD, FusarPoli P, Radua J, et al. Potential reporting bias in fMRI studies of the brain. PLoS ONE. 2013; 8(7):e70104.

37 Ioannidis JP, Munafò MR, Fusar-Poli P, Nosek BA, David SP. Publication and other reporting biases in cognitive sciences: detection, prevalence, and prevention. Trends Cogn Sci. 2014 May;18(5):235-41.

38 Everitt BJ, Robbins TW. Drug addiction: updating actions to habits to compulsions ten years on. Annu Rev Psychol. 2016;67:23-50.

39 Almeida I, Soares SC, Castelo-Branco M. The distinct role of the amygdala, superior colliculus and pulvinar in processing of central and peripheral snakes. PLoS ONE. 2015;10(6): e0129949.

40 Oei JL, Kingsbury A, Dhawan A, Burns L, Feller JM, Clews S, et al. Amphetamines, the pregnant woman and her children: A review. J Perinatology. 2012;32(10):737-47.

41 Oei NYL, Both S, van Heemst D, van der Grond J. Acute stress-induced cortisol elevations mediate reward system activity during subconscious processing of sexual stimuli. Psychoneuroendocrinology. 2014 Jan;39: 111-20.

42 Pessiglione M, Petrovic P, Daunizeau J, Palminteri S, Dolan RJ, Frith CD. Subliminal instrumental conditioning demonstrated in the human brain. Neuron. 2008 Aug 28;59(4): 561-7.

43 Wilcox CE, Pommy JM, Adinoff B. Neural circuitry of impaired emotion regulation in substance use disorders. Am J Psychiatry. 2016 Apr 1;173(4):344-61.

44 Courtney KE, Ghahremani DG, Ray LA. The effects of pharmacological opioid blockade on neural measures of drug cue-reactivity in humans. Neuropsychopharmacology. 2016; 41(12):2872-81.

45 Kilts CD, Gross RE, Ely TD, Drexler KP. The neural correlates of cue-induced craving in cocaine-dependent women. Am J Psychiatry. 2004 Feb;161(2):233-41. 